\title{
Clinical surveillance of the Influenza A(H1N1)2009 pandemic through the network of Sentinel General Practitioners
}

\author{
by \\ Van Casteren $\mathrm{V}^{1}$, Mertens $\mathrm{K}^{1}$, Antoine $\mathrm{J}^{1}$, Wanyama $\mathrm{S}^{1}$, Thomas $\mathrm{I}^{1}$, Bossuyt $\mathrm{N}^{1}$
}

\section{Keywords}

Sentinel surveillance, family practice, Influenza, Influenza A virus, H1N1 Subtype

\section{Introduction}

Since the 1950s, sentinel surveillance networks with general practitioners (GPs) have been progressively implemented and involved in influenza surveillance, e.g. in the UK, the Netherlands, Belgium, France, Portugal and Spain. Since 1988, several collaborative European studies with sentinel networks aimed at stimulating influenza surveillance all over Europe and harmonising the surveillance activities (1-3). In Belgium, a sentinel network specifically dedicated to influenza surveillance, with the participation of about 40 GPs, was initiated in 1985 $(4 ; 5)$ and has participated in various European influenza related projects. Since autumn 2007, this network is integrated into the network of the Sentinel General Practitioners (SGPs) existing since 1979 and responsible for the surveillance of many other health problems (6-8). Since then, the SGPs have been continuously involved in the clinical and virological influenza surveillance (9). As the SGPs network is not appropriate for the detection of sporadic cases, during the containment phase of the $\mathrm{A}(\mathrm{H} 1 \mathrm{~N} 1) 2009$ (from week 14 till week 28), a comprehensive surveillance of all suspected cases of $A(H 1 N 1) 2009$ virus among travellers returning from the USA, Mexico or other countries at risk, was carried out by all Belgian physicians. As of week 29, the start of the mitigation phase, the SGPs were the most important tool for the clinical as well as the virological influenza surveillance. The latter was reinforced with the purpose of following up the $\mathrm{A}(\mathrm{H} 1 \mathrm{~N} 1) 2009$ virus circulation in the general population (10).

The main purposes of this influenza surveillance are the early detection of an influenza epidemic, the study of the intensity and duration of the epidemic and the identification of the circulating viruses. The virological surveillance component is outlined elsewhere in this issue (10). This article details the clinical surveillance activities of the SGPs from week 14, at the appearance of the $A(H 1 N 1) 2009$ virus, until week 53 of 2009. 


\section{Method}

The SGPs are a nationwide sentinel surveillance network of about 160 voluntarily participating GPs homogeneously spread over the country. They are as representative as possible of all GPs in Belgium (11). Besides the number of acute respiratory infections by age group, the GPs reported weekly, on a standardised paper form, every patient with an influenza-like illness (ILI). The general criteria for ILI were: sudden onset of symptoms, high fever, respiratory (i.e. cough, sore throat) and systemic symptoms (headache, muscular pain). For every patient, age group $(<5,5-14,15-64,65-84,85+)$, hospitalisation, antiviral treatment (as of week 35 ), delivery of absence from work certificate, and seasonal and pandemic vaccination status (as of week 42) were recorded.

Reported ILIs were analysed on a weekly basis for inclusion in the "Weekly Epidemiological Report Influenza" (12). This included the weekly reported incidence of ILI for the whole country, the three regions and separate age groups, as well as the weekly reported incidence of ILI-related hospitalisations, of ILI patients who have had antiviral treatment or have been vaccinated for seasonal flu for separate age groups and of ILI patients for whom work certificates were provided. The estimated number of $A(H 1 N 1) 2009$ related cases in the general population was also included in the weekly report. The latter was derived from the percentage of $\mathrm{A}(\mathrm{H} 1 \mathrm{~N} 1) 2009$-positive ILI cases. Data management, statistical analysis and reporting were carried out by the Scientific Institute of Public Health (WIV-ISP), using STATA Version 10 and LaTeX Project software.

Data on vaccination status and work certificates are not presented in this article.

For practical reasons, the data of week 52 and week 53 were analysed together.

Since no patient lists per GP exist in Belgium, the average population coverage per GP was estimated on the basis of the total Belgian population, divided by the total number of practising GPs in the country. The latter was based on figures from the National Institute of Sickness and Invalidity Insurance (NISII) (11). Thus, the average population coverage per GP was estimated at 953 inhabitants per GP. The weekly global population coverage was derived from the total number of participating GPs multiplied by 953. The age distribution of this estimated population was assumed to be similar to the national population.

A baseline threshold was calculated using the Moving Epidemics Method (MEM) proposed by the European Influenza Surveillance Scheme (EISS) baseline working group (13). In principle, each influenza season is mathematically divided into a pre-epidemic, epidemic and a post-epidemic period. The proposed baseline threshold is then calculated, i.e. the upper $95 \%$ confidence limit of the geometric mean of the peak pre-epidemic values. The baseline threshold calculation was based on 5 peak values per influenza season, in 9 historical seasons 2000/01-2008/09. The baseline threshold is a conservative value that demarcates the start of the influenza epidemic. When the weekly incidence rate drops below the postepidemic baseline, the epidemic period is considered to have come to an end. For practical purposes, the pre- and post-epidemic baseline values were considered the same. Based on 
this model and using two-sided tests, the baseline threshold was estimated at 141.37 ILI patients seen in general practice per 100,000 inhabitants per week.

In the framework of this surveillance, the criteria used to define the epidemic influenza period were: weekly incidence rate of ILI patients seen in general practice above the baseline threshold; >20\% positive specimens among nasopharyngeal swabs taken in ILI patients; and signs of influenza activity in neighbouring countries.

\section{Results}

In the period from week 14 until week 53, between 111 and 162 GPs participated in the weekly clinical influenza surveillance. The weekly incidence of ILI patients seen in general practice per 100,000 inhabitants exceeded the baseline threshold for the first time in week 40 (beginning of October 2009) with an incidence of 173/100,000 inhabitants (95\% Cl: 152-196). The incidence rate remained above the baseline threshold until week 49 (at the beginning of December). According to the above-mentioned criteria the influenza epidemic period lasted 10 weeks, from the beginning of October until the beginning of December. The epidemic peaked in week 44 , with an incidence of $769 / 100,000$ inhabitants $(95 \% \mathrm{Cl}: 725-815)$. This peak value is significantly lower $(p<0.05)$ than the $A(H 3 N 2)$ seasonal peak in week 4 of 2009 (844/100,000 inhabitants $(95 \% \mathrm{Cl}: 808-880)$. The peak value in week 44 coincided with a peak in the percentage of $A(H 1 N 1) 2009$ virus-positive swabs (10). Based on this percentage the extrapolated number of $\mathrm{A}(\mathrm{H} 1 \mathrm{~N} 1) 2009$ cases in the general population in week 44 was estimated at $46,848(95 \% \mathrm{Cl}: 43,331-50,641)$.

Figure 1. Sentinel General Practitioners, incidence of ILI patients in general practice, 2009

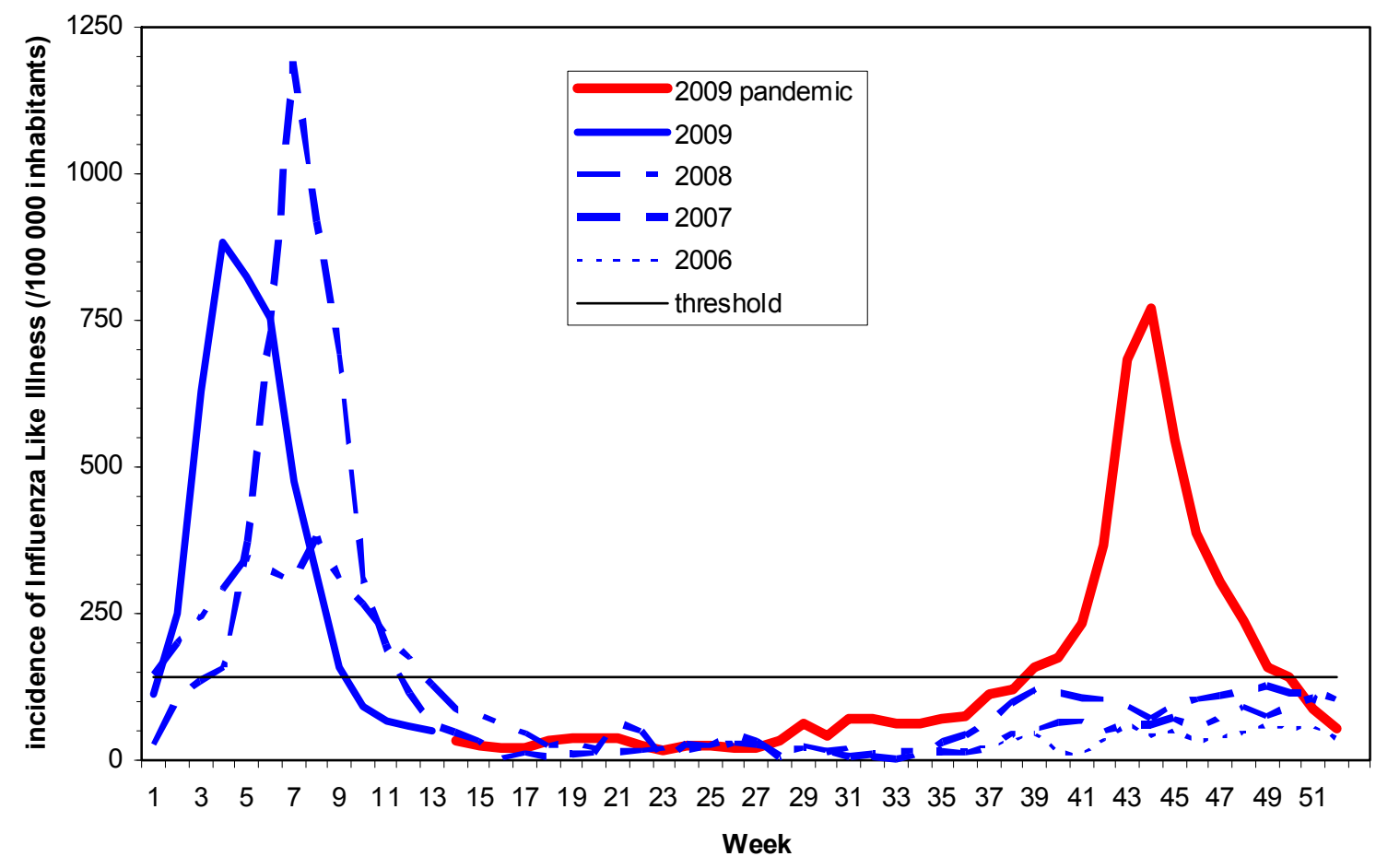


In the course of this epidemic period, the most affected age group was the 5-14-year olds, followed by the $<5$-year olds. People aged 65 years and above were considerably less affected.

Figure 2. Sentinel General Practitioners, incidence of ILI patients in general practice by age group, 2009
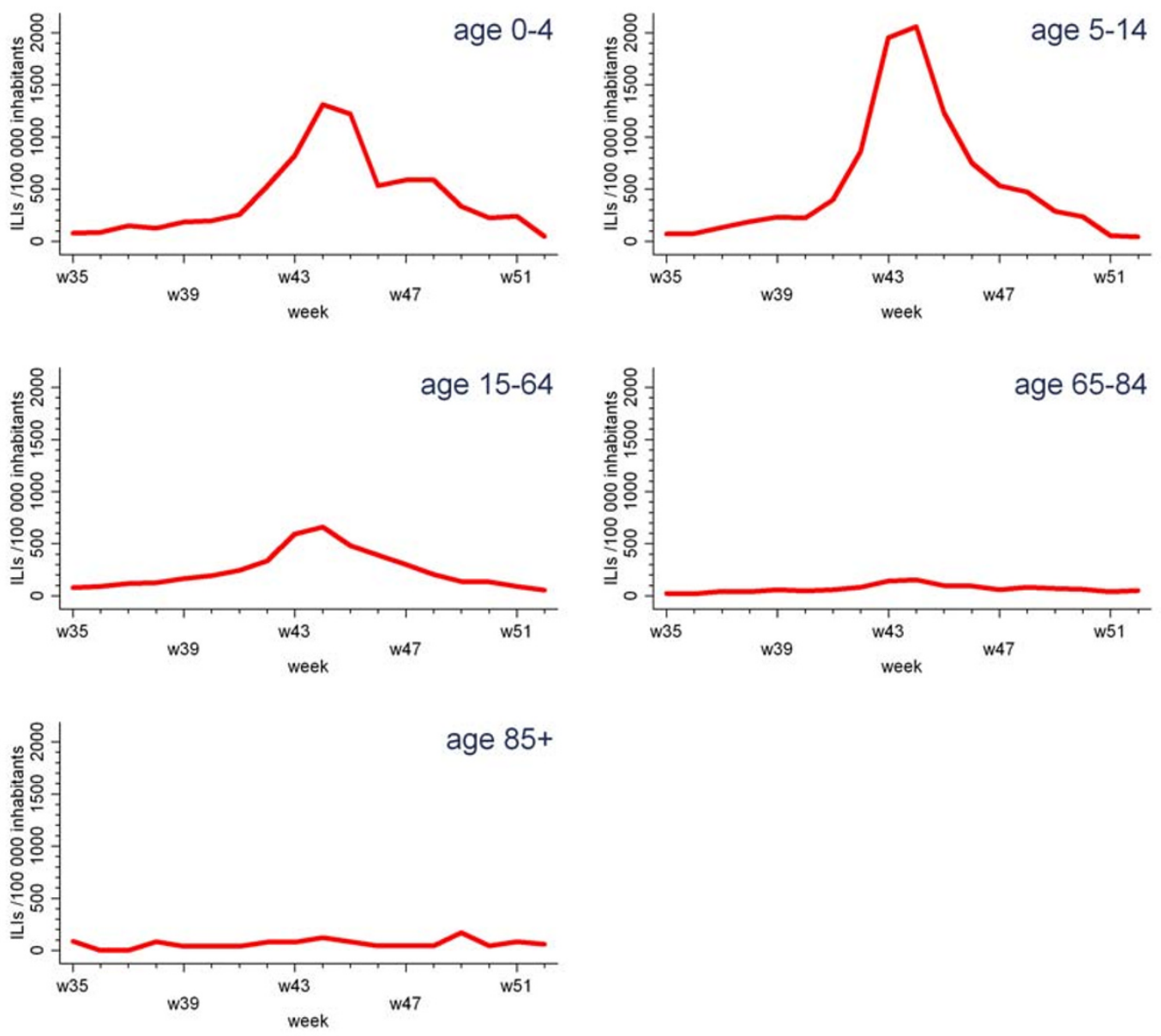

In the course of the epidemic period between week 40 and 49 , the weekly hospitalisation rate for ILI patients seen in general practice fluctuated between 0 and $2 \%$.

The weekly percentage of ILI patients with antiviral treatment, recorded as of week 35 , varied between 1 and $13 \%$.

\section{Discussion}

The network of SGPs has proven to be an important source of information in the influenza surveillance of the $A(H 1 N 1) 2009$. The SGPs provided information on the start, the duration and the peak of the epidemic period. Most of the affected persons were children aged $<15$ years. Few ILI patients seen in general practice were hospitalised or got antiviral treatment. 
Table 1. Sentinel General Practitioners, ILI patients with antiviral treatment by age group, 2009

\begin{tabular}{|c|c|c|c|c|c|c|c|c|c|c|c|c|c|c|c|c|c|c|}
\hline \multirow[t]{2}{*}{ week } & \multicolumn{3}{|c|}{$0-4$} & \multicolumn{3}{|c|}{$5-14$} & \multicolumn{3}{|c|}{$15-64$} & \multicolumn{3}{|c|}{$65-84$} & \multicolumn{3}{|c|}{$85+$} & \multicolumn{3}{|c|}{ tot } \\
\hline & \# & $\# \mathrm{t}$ & $\%$ & $\#$ & $\# \mathrm{t}$ & $\%$ & $\#$ & $\# \mathrm{t}$ & $\%$ & $\#$ & $\# \mathrm{t}$ & $\%$ & \# & $\# \mathrm{t}$ & $\%$ & $\#$ & $\# \mathrm{t}$ & $\%$ \\
\hline $2009 w 35$ & 6 & 0 & 0 & 11 & 0 & 0 & 71 & 1 & 1 & 5 & 0 & 0 & 2 & 0 & 0 & 95 & 1 & 1 \\
\hline $2009 w 36$ & 7 & 0 & 0 & 12 & 1 & 8 & 84 & 10 & 12 & 4 & 1 & 25 & 0 & 0 & & 107 & 12 & 11 \\
\hline $2009 w 37$ & 12 & 1 & 8 & 22 & 0 & 0 & 115 & 6 & 5 & 10 & 1 & 10 & 0 & 0 & & 159 & 8 & 5 \\
\hline $2009 w 38$ & 10 & 1 & 10 & 31 & 2 & 6 & 120 & 7 & 6 & 9 & 1 & 11 & 2 & 0 & 0 & 172 & 11 & 6 \\
\hline $2009 w 39$ & 15 & 1 & 7 & 39 & 0 & 0 & 159 & 8 & 5 & 13 & 2 & 15 & 1 & 0 & 0 & 227 & 11 & 5 \\
\hline $2009 w 40$ & 16 & 0 & 0 & 38 & 0 & 0 & 188 & 9 & 5 & 11 & 2 & 18 & 1 & 0 & 0 & 254 & 11 & 4 \\
\hline $2009 w 41$ & 21 & 3 & 14 & 67 & 1 & 1 & 238 & 19 & 8 & 13 & 3 & 23 & 1 & 0 & 0 & 340 & 26 & 8 \\
\hline $2009 w 42$ & 45 & 8 & 18 & 151 & 5 & 3 & 340 & 22 & 6 & 20 & 2 & 10 & 2 & 0 & 0 & 558 & 37 & 7 \\
\hline $2009 w 43$ & 67 & 5 & 7 & 328 & 27 & 8 & 573 & 61 & 11 & 32 & 4 & 13 & 2 & 0 & 0 & 1002 & 97 & 10 \\
\hline $2009 w 44$ & 107 & 8 & 7 & 346 & 9 & 3 & 637 & 39 & 6 & 35 & 3 & 9 & 3 & 0 & 0 & 1128 & 59 & 5 \\
\hline $2009 w 45$ & 97 & 4 & 4 & 202 & 5 & 2 & 456 & 26 & 6 & 22 & 4 & 18 & 2 & 1 & 50 & 779 & 40 & 5 \\
\hline $2009 w 46$ & 40 & 5 & 13 & 116 & 4 & 3 & 348 & 18 & 5 & 20 & 3 & 15 & 1 & 0 & 0 & 525 & 30 & 6 \\
\hline $2009 w 47$ & 44 & 6 & 14 & 82 & 3 & 4 & 265 & 11 & 4 & 12 & 0 & 0 & 1 & 0 & 0 & 405 & 20 & 5 \\
\hline $2009 w 48$ & 46 & 0 & 0 & 76 & 3 & 4 & 191 & 9 & 5 & 18 & 5 & 28 & 1 & 0 & 0 & 332 & 17 & 5 \\
\hline $2009 w 49$ & 26 & 2 & 8 & 46 & 1 & 2 & 127 & 4 & 3 & 15 & 1 & 7 & 4 & 0 & 0 & 219 & 8 & 4 \\
\hline $2009 w 50$ & 17 & 2 & 12 & 38 & 0 & 0 & 122 & 6 & 5 & 13 & 1 & 8 & 1 & 0 & 0 & 191 & 9 & 5 \\
\hline $2009 w 51$ & 19 & 4 & 21 & 9 & 1 & 11 & 85 & 11 & 13 & 9 & 0 & 0 & 2 & 0 & 0 & 124 & 16 & 13 \\
\hline $2009 w 52$ & 6 & 0 & 0 & 10 & 0 & 0 & 89 & 2 & 2 & 31 & 2 & 6 & 2 & 0 & 0 & 138 & 4 & 3 \\
\hline
\end{tabular}

\#=number of ILIs reported; \#t=number of ILI-patients treated with antivirals reported; \%=percentage of ILI-patients treated with antivirals (\#/100 ILIs)

Strengths of the SGPs' influenza surveillance

The SGPs have a long experience in public health surveillance. The highly motivated participating GPs are a guarantee for the quality of the participation and of the recorded data. The clinical influenza surveillance data are corroborated by the virological data originating from the same patients (10). The continuous weekly influenza monitoring year after year enables to respond to the influenza surveillance purposes previously outlined and to compare consecutive influenza seasons as to the intensity and duration of the epidemics and the type of circulating viruses.

\section{Weaknesses}

One weakness is the denominator problem caused by the absence of patient lists per GP. Only a crude estimation of the denominator population can be made. Further, sensitivity or completeness of the ILI recording is hampered by the fact that the surveillance only concerns patients visiting the GP and does not include ILI patients visiting other health care providers or not consulting at all. Both weaknesses, however, do not hamper studying the trend in ILI incidence. Finally, the weekly reporting frequency hinders the daily follow-up of the situation, desirable in an epidemic period.

This does not refrain the SGPs from playing a crucial role in the clinical as well as in the virological influenza surveillance in this $A(H 1 N 1) 2009$ pandemic. 


\section{Acknowledgments}

The network of SGPs is financially supported by the Flemish and French Community of Belgium. The authors would like to thank all GPs that contribute to this influenza surveillance.

\section{References}

1. Paget J, Marquet R, Meijer A, Van der Velden K. Influenza activity in Europe during eight seasons (1999-2007): an evaluation of the indicators used to measure activity and an assessment of the timing, length and course of peak activity (spread) across Europe. BMC Infect Dis 2007; 7:141

2. Snacken $R$, Bensadon $M$, Strauss $A$. The CARE telematics network for the surveillance of influenza in Europe. Methods Inf Med 1995; 34(5):518-22

3. Van Casteren V, Leurquin P. Eurosentinel: development of an international sentinel network of general practitioners. Methods Inf Med 1992;31(2):147-52

4. Snacken R, Lion J, Van C, V, Cornelis R, Yane F, Mombaerts $M$ et al. Five years of sentinel surveillance of acute respiratory infections (1985-1990): the benefits of an influenza early warning system. Eur J Epidemiol 1992; 8(4):485-90

5. Snacken R. Weekly monitoring of influenza impact in Belgium (1993-1995). Pharmacoeconomics 1996; 9 Suppl 3:34-7

6. Lobet MP, Stroobant A, Mertens R, Van C, V, Walckiers D, Masuy-Stroobant G et al. Tool for validation of the network of sentinel general practitioners in the Belgian health care system. Int J Epidemiol 1987; 16(4):612-18

7. Stroobant A, Van Casteren V, Thiers G. Surveillance systems from primary-care data : surveillance through a network of sentinel general practitioners. In: Eylenbosch W, Noah D, editors. Surveillance in Health en Disease. Oxford: Oxford University Press, 1988: 62-74

8. Van Casteren V. Thirty years Registration Network of Sentinel General Practitioners. Arch Public Health 2009; 67(S2):3-15

9. Thomas I, Van Casteren V. Surveillance de la Grippe saisonnière en Belgique. Saison 2007-2008. Bruxelles: ISP-WIV; 2009. D/2505/2008/56

10. Gérard C, Brochier B, Quoilin S, Wuillaume Françoise, Van Casteren V, Thomas I. Virological surveillance of Influenza $\mathrm{A}(\mathrm{H} 1 \mathrm{~N} 1) 2009$ pandemic: the roles of the Belgian National Influenza Centre. Arch Public Health 2010; 68(2):68-75

11. Boffin N, Bossuyt N, Van Casteren V. Caractéristiques des Médecins Vigies et de leur pratique. Situation en 2007 et comparaison avec les années précédentes. Bruxelles: ISP-WIV; 2009. D/2009/2505/17.

12. Scientific Institute of Public Health. Influenza. [Online]. 2010 [cited 2010 Jan 27]. Available from: URL: http://www.wiv-isp.be/flu/EN/Y2009-Influenza.pdf

13. Vega Alonso T Lozano Alonso JE, Ortiz de Lejaraza R, Gutierrez Perez MS. Modelling influenza epidemic - can we detect the beginning and predict the intensity and duration? International Congress Series $2004 ; 1263: 281-83$ 